\title{
Vessel wall imaging in intracranial aneurysms
}

\author{
Edgar A Samaniego (1) , ${ }^{1}$ Jorge A Roa, ${ }^{2}$ David Hasan ${ }^{3}$
}

${ }^{1}$ Neurology, Neurosurgery and Radiology, University of lowa Hospitals and Clinics, lowa City, lowa, USA

${ }^{2}$ Neurology and Neurosurgery, University of lowa Hospitals and Clinics, lowa City, lowa, USA ${ }^{3}$ Neurosurgery, University of lowa Hospitals and Clinics, lowa City, lowa, USA

\section{Correspondence to}

Dr Edgar A Samaniego, Interventional Neuroradiology/ Endovascular Neurosurgery Division Department of Neurology, Neurosurgery and Radiology, University of lowa, lowa City, IA 52242, USA; edgarsama@gmail.com

Received 1 April 2019 Revised 30 May 2019 Accepted 5 June 2019 Published Online First 23 July 2019

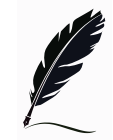

This article was commissioned.

(C) Author(s) (or their employer(s)) 2019. No commercial re-use. See rights and permissions. Published by BMJ.

To cite: Samaniego EA,

Roa JA, Hasan D.

J Neurolntervent Surg

2019:11:1105-1112.

\section{ABSTRACT}

High-resolution vessel wall imaging (HR-VWI) is becoming a useful tool in the characterization and identification of unstable unruptured brain aneurysms. However, it has not been validated for clinical use. The current evidence on HR-VWI techniques for characterization of brain aneurysms is described in this review. Specific imaging approaches such as aneurysm wall contrast enhancement, MRI-quantitative susceptibility mapping, and $7 \mathrm{~T} \mathrm{MRI} \mathrm{are} \mathrm{described} \mathrm{in}$ detail.

\section{INTRODUCTION}

Unruptured intracranial aneurysms (UIAs) are found in $3-5 \%$ of the adult population worldwide. The large majority of UIAs will never rupture; however, $27 \%$ of patients who develop aneurysmal subarachnoid hemorrhage (aSAH) may die within 12 months. $^{2}$ Early recognition of aneurysms with high rupture risk is crucial in stratification. Scores such as the PHASES score created to predict a patient's 5-year risk of aneurysm rupture based on patient characteristics and aneurysm morphology are not routinely used in clinical practice. ${ }^{3}$ Moreover, aneurysm characteristics may vary among different regions of the world, ${ }^{4}$ limiting the use of scores that have not been validated worldwide.

High-resolution vessel wall imaging (HR-VWI) has emerged as a valuable tool in assessing unstable UIAs. There are promising observations in the characterization of aneurysm wall enhancement as a biomarker of aneurysm wall inflammation, aneurysm growth and rupture. ${ }^{5}$ This review aims to summarize the current information about HR-VWI in cerebral aneurysm characterization and to reflect our experience.

\section{ANEURYSM WALL INFLAMMATION}

Different theories have incorporated a combined explanation for intracranial aneurysm formation that includes hemodynamic stress, endothelial dysfunction, and inflammation. ${ }^{6}$ Virchow first suggested inflammation as a cause of intracranial aneurysms in $1847 .{ }^{7}$ Other indirect causes of vessel wall inflammation that may lead to aneurysm formation include oncotic infiltration of the vessel wall, such as in the case of myxomatous aneurysms (figure 1), or formation of mycotic aneurysms due to the presence of microorganisms. ${ }^{89}$

Chyatte et al reported that inflammation and immunological reactions are common features of UIAs. ${ }^{10}$ Higher levels of complement, IgG, IgM, macrophages, and T lymphocytes were observed in the aneurysm wall compared with normal vessels.
Since then, several studies have suggested specific immunological pathways and mediators for aneurysm formation and rupture. ${ }^{911}$

Prolonged inflammatory changes may lead to continued wall remodeling and ultimately to atherosclerotic changes with accumulation of low-density lipoprotein. In the majority of aneurysms rupture happens at the fundus. This area of the sac is usually infiltrated by polymorphonuclear cells and has fibrotic changes. ${ }^{12}$

\section{ANEURYSM WALL ENHANCEMENT AS AN INDICATOR OF ANEURYSM INSTABILITY}

Several studies have suggested that aneurysmal wall enhancement on HR-VWI might help in identifying UIAs with a higher risk of rupture (table 1). Grading methods for aneurysmal wall enhancement have been proposed, mainly based on a subjective appreciation of wall enhancement and different objective methods to quantify signal intensity. The subjective appreciation divides enhancement on: strong/ avid, mild, and no wall enhancement. Most of the studies that quantify wall enhancement subjectively describe blinded adjudication by two or three experienced neuroradiologists. The objective methods measure focal aneurysmal wall enhancement and circumferential aneurysmal wall enhancement (CAWE) with post-contrast quantification of signal intensity. An enhancement ratio may be generated between signal intensity sampling at the aneurysmal sac and the pituitary stalk, or between pre- and post-contrast T1-weighted images (figures 2 and 3).

In order to establish a relationship between imaging findings and histopathological changes of the aneurysm wall, ex vivo specimens have been analyzed by different groups. Hu et al described one patient with a ruptured middle cerebral aneurysm (MCA) who underwent clipping and histological analysis. HR-VWI demonstrated avid wall enhancement of the aneurysm that correlated with lymphocyte and phagocyte infiltration. ${ }^{13}$ Larsen et al analyzed 13 UIAs located in the MCA. ${ }^{14}$ Five aneurysms showed strong wall enhancement and had some degree of myeloperoxidase (MPO) activity, neovascularization or presence of vasa vasorum. Shimonaga et al correlated HR-VWI of nine UIAs with histological analysis. Five aneurysms had wall enhancement and pathological findings: vasa vasorum disease, neovascularization, and macrophage infiltration. ${ }^{15}$ Similarly, our group analyzed 10 wall specimens from patients with UIAs. Five UIAs with avid enhancement on HR-VWI had higher average wall thickness $(p=0.003)$, macrophage infiltration and cellularity $(p=0.048)$ compared with aneurysms with mild- or no-enhancing wall. Of note, all tissue samples in our 


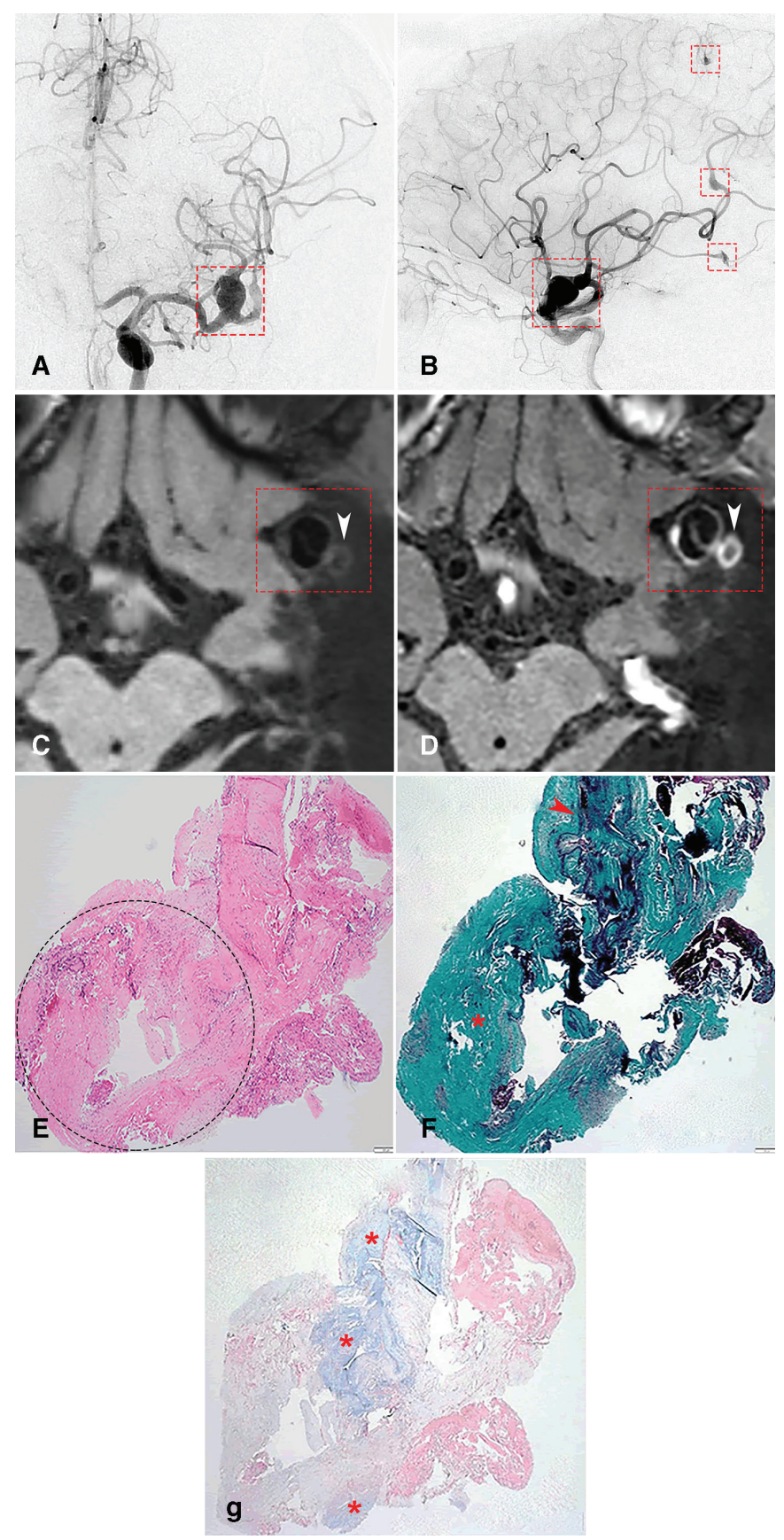

Figure 1 (A) Anterior and (B) lateral digital substraction angiography (DSA) in a patient with multiple myxomatous aneurysms (red squares). (C) 3 T axial T1-weighted pre- and (D) post-gadolinium images showing partial circumferential aneurysm wall enhancement (CAWE) of the MCA aneurysm depicted in DSA (red square) and complete CAWE of the daughter sac (white arrowheads). (E) Hematoxylin-eosin stain of pathological specimen (40x) from the aneurysm and its daughter sac (dotted ellipse). (F) Elastichrome stain demonstrating loss of elastic lamina in the daughter sac (red asterisk) compared with the dome of the aneurysm (red arrowhead). (G) Alcian Blue stain demonstrating myxoid intimal degeneration of both the dome and daughter sac of the aneurysm (red asterisks).

series were deficient in elastin. ${ }^{16}$ More recently, Matsushige et al reported on four aneurysms imaged with a $1.5 \mathrm{~T}$ scan, three with focal enhancement and one with CAWE. The aneurysms with focal enhancement had thin walls $(20-50 \mu \mathrm{m})$ compared with aneurysms with CAWE, which had thick walls $(120-320 \mu \mathrm{m})$ and decreased cellularity and elastic fibers as well as the presence of neovascularization. ${ }^{17}$ Quan et al also reported on nine UIAs that were analyzed after clipping. ${ }^{18}$ Decreased endothelium and elastic lamina were described in enhancing $(n=6)$ and non-enhancing aneurysms $(n=3)$. Aneurysms with focal enhancement exhibited more atherosclerotic burden than aneurysms with CAWE. To date, these are the only six published studies that have confirmed wall enhancement on HR-VWI with the presence of inflammatory changes on histopathological specimens. However, the inflammatory changes described in these studies are not uniform. Vessel wall enhancement possibly occurs with various types of histologic changes, likely representing different stages and phenotypes of vasculopathy, leading to aneurysm formation and growth. ${ }^{419}$ It is also difficult to compare these studies as there is large heterogeneity in the segment of the aneurysm sampled for histopathological analysis (dome, neck, bleb) and in the processing of the sample. However, it appears that common denominators in the histopathological analysis of these aneurysms are the presence of active macrophages, neovascularization, and decreased elastin.

Some studies have demonstrated an association between wall enhancement and known clinical risk factors of aneurysm rupture. Lv et al studied 110 patients with UIAs and showed a significant relationship between HR-VWI wall enhancement and larger size $(\geq 7 \mathrm{~mm})$ and location in the anterior cerebral, posterior communicating, and posterior circulation arteries $(\mathrm{p}<0.001) .{ }^{20}$ Recently, Hartman et al compared wall enhancement of 65 UIAs (45 patients) imaged with 3 T HR-VWI and their PHASES scores. Aneurysms with a PHASES score $>3$ were more likely to demonstrate wall enhancement (42.1\% vs $14.8 \%$, $\mathrm{p}=0.022)$ and wall thinning $(9.2 \%$ vs $0 \%, \mathrm{p}=0.044) .{ }^{21}$ Of note, nine patients $(20 \%)$ in this study were scanned using the volume isotropic turbo-spin-echo acquisition (VISTA) protocol, which might be highly susceptible to flow artifacts and aneurysm wall thickness overestimation. ${ }^{22}$ These two studies used PHASES score criteria to determine the risk of rupture of the aneurysm. However, as the authors underlined, the PHASES score does not account for irregular aneurysm shape, which has a strong correlation with risk of rupture.

Backes et al studied the determinants of wall enhancement in 89 predominantly small UIAs $(84 \%<7 \mathrm{~mm})$. The strongest determinant of aneurysm wall enhancement was aneurysm size, followed by posterior communicating artery or MCA location. Larger aneurysms showed more enhancement: RR 4.6 (95\% CI 0.6 to 36.5 ) for aneurysms $1-2.9 \mathrm{~mm}$ compared with RR 14.8 (95\% CI 2.1 to 104.6$)$ for aneurysms $\geq 7 \mathrm{~mm}$. Interestingly, there was no association between aneurysm wall enhancement and smoking status, use of acetylsalicylic acid or statins, hypertension and aneurysms with irregular shape. ${ }^{23}$ Another study by Liu et al that included 48 patients with 61 UIAs also found that aneurysm size was independently associated with aneurysm wall enhancement. ${ }^{24}$ However, in contrast with the study by Backes et al, Liu's study included predominantly large aneurysms $(59 \% \geq 7 \mathrm{~mm})$. Recently, Wang and colleagues analyzed 88 UIAs and demonstrated that irregular shape and high depth/neck width aspect ratio were significantly associated with wall enhancement on HR-VWI (OR 12.5, $\mathrm{p}=0.02$ and OR 32.9, $\mathrm{p}=0.01$, respectively).$^{25}$ Although these observational studies have associated aneurysm size and shape with enhancement on HR-VWI, computational flow dynamics data have suggested that the increased turbulent blood flow within larger and more irregular aneurysms might induce contrast stagnation along the vessel wall and concomitant pseudo-enhancement (figure 4). ${ }^{26}$ 


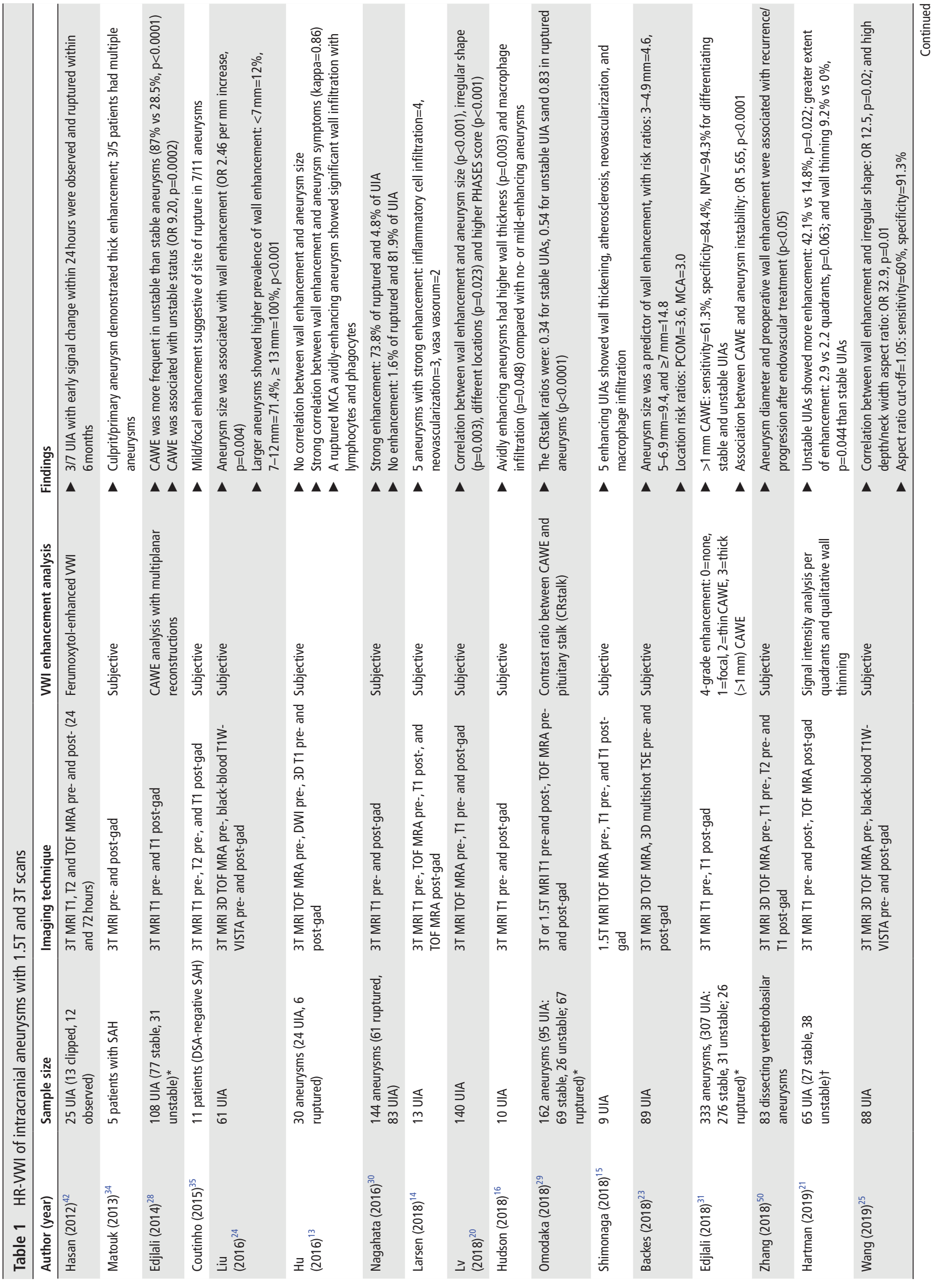



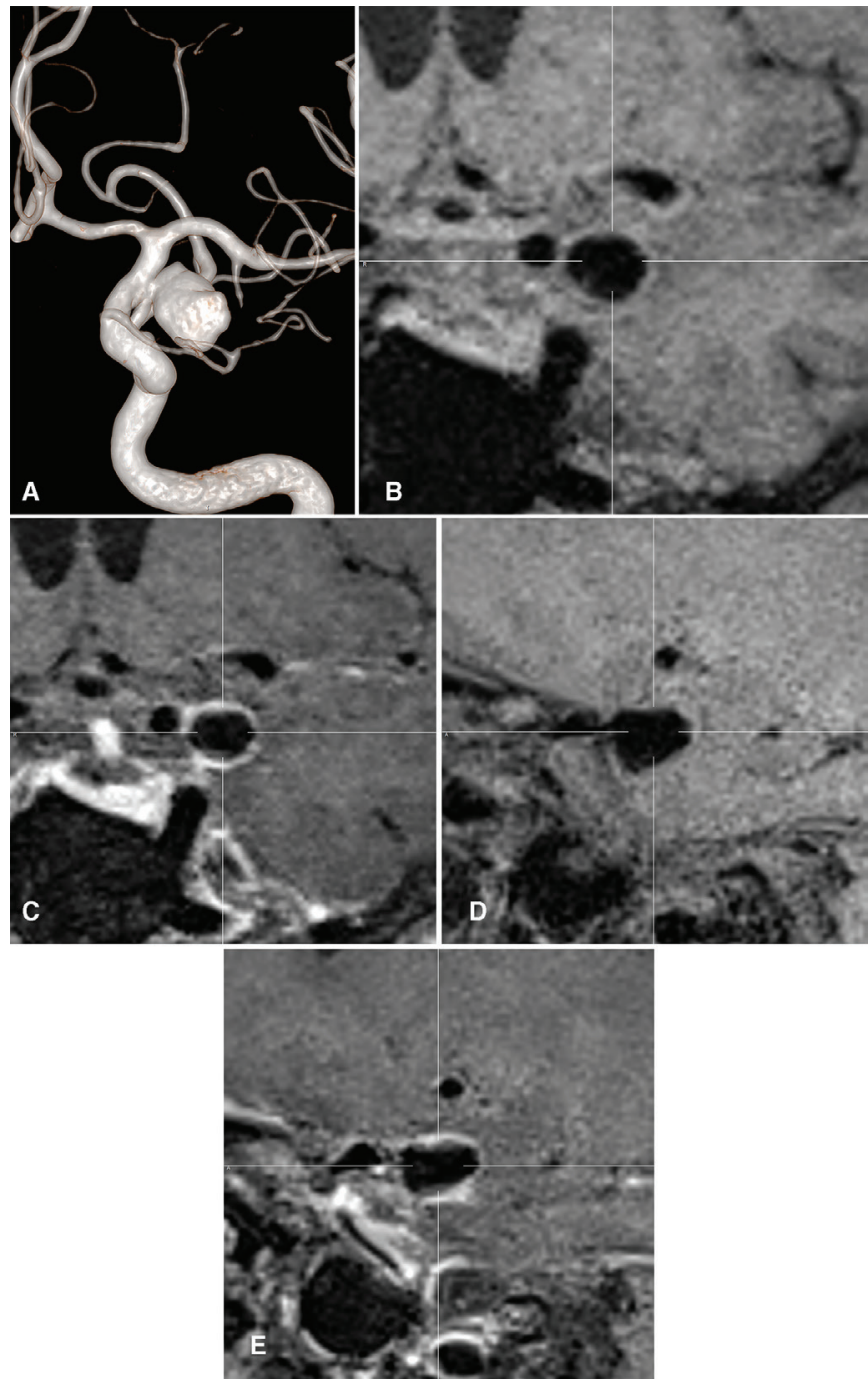

Figure 2 (A) 3D rotational angiogram of a left internal carotid artery supraclinoid segment aneurysm. 7T HR-VWI of the aneurysm demonstrating T1-weighted images of the aneurysm in coronal ( $B$ and $C$ ) and sagittal projections ( $D$ and $E$ ). Notice the presence of avid circumferential aneurysm wall enhancement (CAWE) in the post gadolinium T1-weighted images ( $C$ and $E$ ) compared with the precontrast T1-weighted images ( $\mathrm{B}$ and $\mathrm{D}$ ).

A thickened and partially thrombosed aneurysmal wall may also exhibit avid enhancement (figure 5). Additionally, larger aneurysms might be more prone to present a contrast leakage phenomenon through the vessel wall not quantified by standard HR-VWI protocols. ${ }^{27}$ Therefore, it is important to analyze these aneurysms in multiple planes and with co-registration of 3D images to clearly map the location and thickness of the aneurysm wall. We are still learning how to identify and interpret these artifacts.

Surrogates of aneurysm instability such as growth over time or mass effect on cranial nerves have also been correlated with increased wall enhancement on HR-VWI. Edjlali and colleagues performed HR-VWI in 87 patients with 108 intracranial aneurysms. Aneurysms that became symptomatic, changed on serial magnetic resonance angiography (MRA), or ruptured were considered unstable. CAWE was more frequently seen in unstable than in stable aneurysms $(27 / 31,87 \%$ vs $22 / 77,28.5 \%$, respectively; $\mathrm{p}<0.0001)$. A multivariate logistic regression analysis showed that the only independent factor associated with instability was 

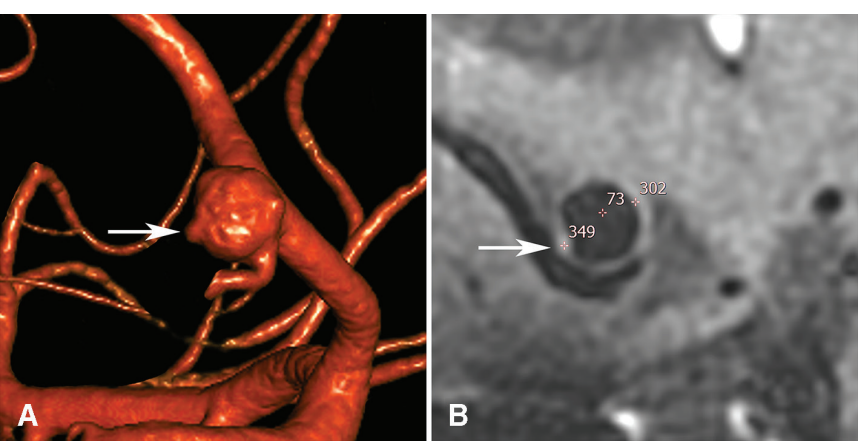

Figure 3 (A) 3D rotational angiogram of a patient with an unruptured anterior communicating aneurysm. The aneurysm has an irregular morphology with multiple blebs. A conventional 3T MRI did not demonstrate irregularities in the aneurysm wall, nor wall enhancement. However, a 7T HR-VWI (B) showed a focal area of contrast enhancement in the area where the most prominent bleb is located ( $A$ and $B$, white arrows). Signal intensity sampling at different locations (bleb $=349$, aneurysm wall $=302$ and aneurysm lumen=73) shows objectively the presence of increased signal intensity at the bleb.

CAWE (OR 9.20; 95\% CI 2.92 to $29.0, p=0.0002) .{ }^{28}$ Of note, 16 of 17 ruptured aneurysms demonstrated CAWE. However, it is unclear if increased enhancement in these aneurysms is a direct result of rupture. Omodaka et al also assessed CAWE, calculating the contrast enhancement ratio between the aneurysmal wall and the pituitary stalk in 26 unstable UIAs, and compared it with ratios from 69 stable UIAs and 67 ruptured aneurysms. The contrast ratio in unstable UIAs was significantly higher than in stable UIAs $(0.54$ vs $0.34, \mathrm{p}<0.0001)$ and significantly lower than in ruptured aneurysms $(0.54$ vs $0.83, \mathrm{p}<0.0002) .{ }^{29}$ In
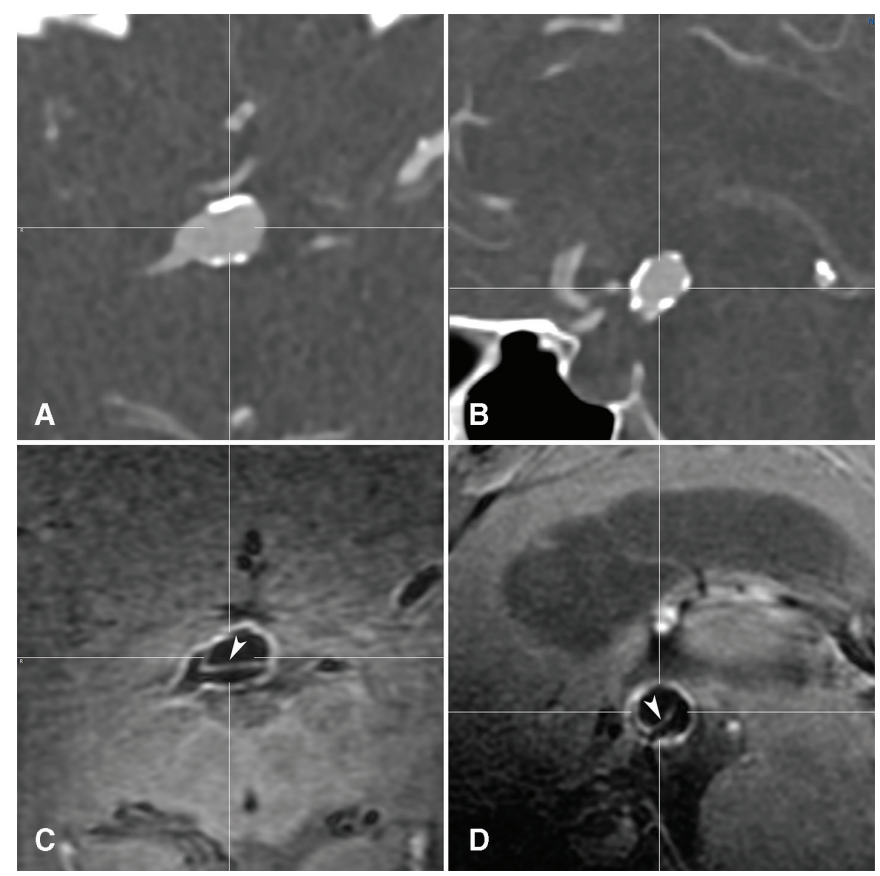

Figure 4 Flow artifact in a basilar tip aneurysm. Coronal $(A)$ and sagittal (B) CT angiography demonstrating a large basilar tip aneurysm with calcified walls. 7T HR-VWI in coronal (C) and sagittal (D) projections depicting an area of enhancement (white arrowheads) in the lumen of the aneurysm, which corresponds to flow artifact. Multiplanar visualization of the aneurysm and co-registration of the time-of-flight sequences are essential in determining the aneurysm wall.
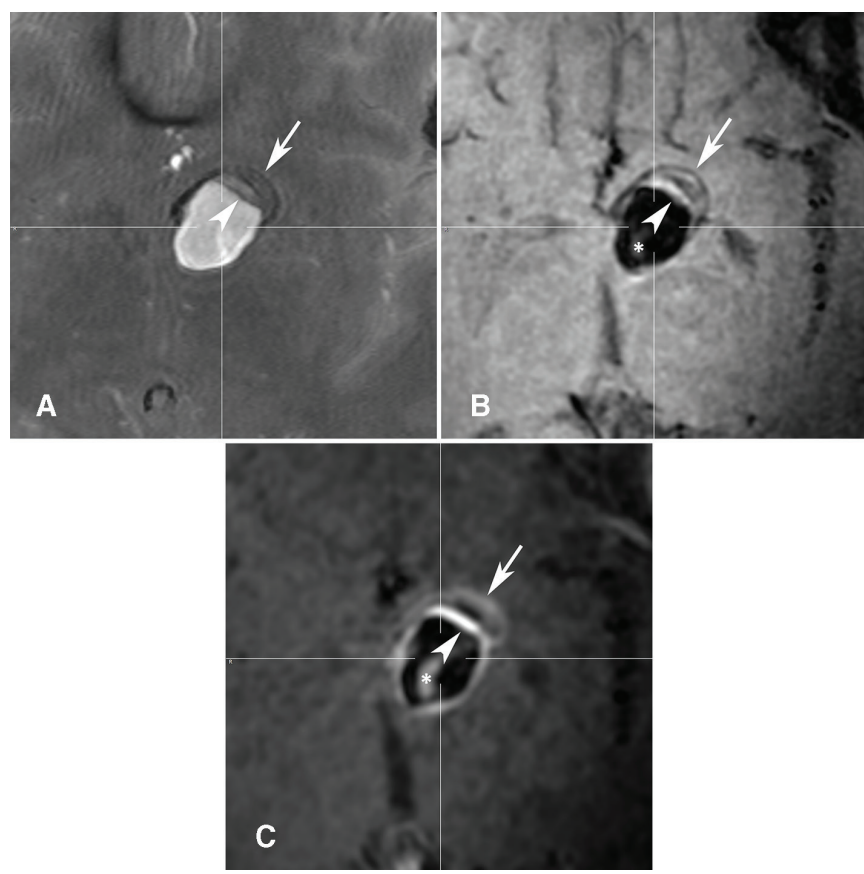

Figure 5 Axial views of a large left carotid terminus aneurysm. (A) Time of flight, (B) pre T1-weighted, and (C) post-gadolinium T1weighted 7T HR-VWI demonstrating thrombus artifact within the aneurysm wall. Avid contrast enhancement (C) of the inner surface of the aneurysm wall (arrowhead) suggests leakage of contrast across the partially thrombosed and thickened wall. The outer surface of the wall (arrow) does not enhance as much as the rest of the aneurysm. Flow artifact (asterisk) is also noticed in the aneurysm lumen.

another study, Nagahata et al retrospectively reviewed HR-VWI findings of 117 patients with 144 aneurysms (61 ruptured and 83 UIAs). Strong wall enhancement was detected in $73.8 \%$ and $4.8 \%$ of ruptured and UIAs, respectively. No enhancement was found in $1.6 \%$ of ruptured and $81.9 \%$ of UIAs. ${ }^{30}$ More recently, Edjlali et al determined CAWE in 263 patients with 333 aneurysms: 307 UIAs (276 stable and 31 unstable) and 26 ruptured aneurysms. CAWE was subjectively classified in four grades: $0=$ none, $1=$ focal, $2=$ thin enhancement, and $3=$ thick $(>1 \mathrm{~mm})$ enhancement. Grade 3 CAWE exhibited the highest specificity $(84.4 \%, 95 \% \mathrm{CI} 80.1 \%$ to $88.7 \%, \mathrm{p}=0.02)$ and negative predictive value $(94.3 \%)$ for differentiating between stable and unstable aneurysms. Multivariate analysis showed a significant association between CAWE and aneurysm instability (OR 5.65, $95 \%$ CI 2.81 to $11.31, \mathrm{p}<0.0001) .{ }^{31}$ Finally, Wang et al analyzed HR-VWI findings of 89 UIAs (31 symptomatic and 58 asymptomatic). An enhancement ratio (ER) was calculated after several signal intensities were measured in the aneurysm neck, body, and dome using T1 pre- and post-gadolinium sequences. A multiple logistic regression analysis showed that ER was associated with symptomatic intracranial aneurysms (OR 29.4, p <0.001). An ER with a threshold value of $60.5 \%$ achieved a sensitivity of $90.3 \%$ and a specificity of $87.9 \% .{ }^{32}$ Overall, these studies suggest that larger aneurysm size, irregular shape, and CAWE are predictors of aneurysm instability due to increased wall enhancement on HR-VWI. However, since most studies included ruptured aneurysms as a surrogate of instability, there is an intrinsic bias in these results that preclude their clinical application in the evaluation of UIAs. Wall enhancement is also arbitrarily assigned by different methods, which limits reproducibility. 


\section{ANEURYSM WALL ENHANCEMENT FOR IDENTIFICATION OF RUPTURED ANEURYSMS}

Accurate non-invasive identification of the source of hemorrhage is essential for targeted treatment in patients with aSAH and multiple aneurysms. Several criteria are used at the time of determining which aneurysm has ruptured: hemorrhage distribution, aneurysm morphology (size, location, shape, neck to dome ratio), filling characteristics, presence of focal vasospasm, and localizing signs and symptoms. ${ }^{33}$

Matouk et al reported the utility of 3T HR-VWI in identifying the site of rupture in patients with aSAH. Three out of five patients had multiple aneurysms. None of the associated UIAs demonstrated thick vessel wall enhancement, which was only visualized in culprit aneurysms as determined by the location and distribution of aSAH on initial non-contrast $\mathrm{CT} .{ }^{34}$ In another study, Coutinho et al used HR-VWI to further characterize 11 patients with spontaneous, angiogram-negative, non-perimesencephalic SAH. Vessel wall enhancement was visualized in seven patients. ${ }^{35}$ HR-VWI demonstrated an area of focal enhancement contiguous with the outer margin of the basilar artery wall in two patients. The authors argue that this area of focal enhancement may represent a thrombosed saccular aneurysm arising from the basilar sidewall or the presence of a blister-like aneurysm. However, this was not confirmed in histology or follow-up imaging.

Aneurysm size alone cannot be used to determine the source of bleeding in patients with aSAH and multiple intracranial aneurysms. It has been shown that up to $\sim 30 \%$ of patients may have a smaller aneurysm that ruptured. ${ }^{36}$ Kondo et al reported a case of aSAH with a $4.2 \mathrm{~mm}$ anterior communicating artery (ACOM) aneurysm and $5.8 \mathrm{~mm}$ basilar tip aneurysm. HR-VWI demonstrated increased enhancement of the smaller ACOM aneurysm. Rupture of the smaller aneurysm was confirmed during microsurgical clipping. ${ }^{37}$ Bhogal et al reported a case of diffuse aSAH and multiple bilateral aneurysms. The largest aneurysm was located on the left MCA bifurcation. HR-VWI showed thick circumferential wall enhancement of a smaller ACOM aneurysm and very faint enhancement of the larger left MCA bifurcation aneurysm. During microsurgical clipping it was confirmed that the ACOM aneurysm ruptured.

Identification of the ruptured aneurysm in patients with multiple intracranial aneurysms may also guide medical management in case of vasospasm. HR-VWI may potentially become a predictive tool in determining stability of the remaining untreated aneurysms and safety of inducing higher blood pressures in the setting of vasospasm. Mossa-Basha et al performed 3T HR-VWI on 29 patients with 30 aneurysms (22 ruptured and 8 UIAs). As expected, ruptured aneurysms significantly showed more enhancement than UIAs: $29.9 \%$ vs $7.2 \%$; OR $5.5,95 \%$ CI 2.2 to 13.7. Moreover, wall enhancement was significantly associated with subsequent angiographic vasospasm while controlling for grade of hemorrhage (adjusted OR 3.9, 95\% CI 1.7 to 9.4), suggesting that aneurysm wall enhancement of ruptured aneurysms may be associated with development of angiographic vasospasm. $^{38}$

To date, two meta-analyses have studied HR-VWI and aneurysm instability or risk of rupture. Both of them included almost the same observational cohorts. Texakalidis et al included six studies comprising 505 saccular aneurysms: 302 stable and 203 unstable (ruptured, growing, or symptomatic). ${ }^{5}$ Aneurysms with wall enhancement had statistically significantly higher odds of instability (OR 20, 95\% CI 6.4 to 62.1). Such relationship remained significant after excluding ruptured aneurysms (OR $10.9,95 \%$ CI 1.66 to 72.62 ). The sensitivity, specificity, positive predictive value, and negative predictive value of HR-VWI in identifying unstable aneurysms were 95\% (90.4-97.8\%), 62.7\% (57.1-67.9\%), 55.8\% (52.2-59.4\%), and 96.2\% (92.8-98\%), respectively, with an overall accuracy of 73.4\% (69.2-77.3\%). These results suggest that no-enhancement on HR-VWI may have a stronger role in excluding high-risk aneurysms than enhancing lesions in predicting instability or risk of rupture. The second meta-analysis by Wang et al included five studies with 402 patients and 492 aneurysms. Wall enhancement was observed in 288 aneurysms: 160 UIAs and 128 ruptured. ${ }^{39}$ This meta-analysis showed a significant positive association between wall enhancement and aneurysm rupture (OR 34.2, 95\% CI 10.2 to $115, \mathrm{p}<0.001) .^{39}$ Again, it is not surprising that ruptured aneurysms enhance more as interrruption of the blood brain barrier may result in contrast leakage.

\section{IRON IMAGING IN THE ANEURYSM WALL}

Macrophages weaken the aneurysm wall by secreting extracellular matrix proteolytic enzymes and generating reactive oxygen species (ROS). ROS may dissociate heme from its protein-bound state, creating pro-oxidative free heme and free iron ions. ${ }^{69}$ Moreover, free hemosiderin, an iron storage complex of erythrocytes, and even intact erythrocytes have been identified in the aneurysm wall. ${ }^{9} 4041$

A pilot study by our group in 22 patients with 30 UIAs showed that circumferential wall uptake of superparamagnetic particles of iron oxide (ferumoxytol) during the first 24 hours after infusion was highly predictive of aneurysm rupture. ${ }^{42}$ As ferumoxytol is cleared by macrophages, visualization of ferumoxytol in the aneurysm wall suggested the presence of active macrophages, which was later confirmed by immunohistological staining. Seven aneurysms $(23 \%)$ showed early uptake of ferumoxytol within 24 hours: Three aneurysms ruptured within 6 months and four aneurysms were clipped. Histological analysis of these aneurysms showed increased expression of M1 macrophages and inflammatory molecules (mPGES- 1 and COX-2) compared with aneurysms with late ferumoxytol uptake $(\mathrm{p}<0.05)$. Nine of 23 aneurysms that had late or no uptake of ferumoxytol did not rupture or change in size or shape after 6 months. These results suggest that early in vivo macrophage uptake of ferumoxytol is a sign of aneurysmal instability due to active macrophage activation. ${ }^{42}$ Honkanen et al analyzed specimens of three giant aneurysms on a $4.7 \mathrm{~T}$ scan. Histology showed a degenerated hypocellular wall with focal luminal, intramural, and adventitial iron accumulation. ${ }^{41}$ The authors argued that high iron accumulation across the aneurysm wall may be associated with increased fragility and risk of rupture.

MRI-quantitative susceptibility mapping (MRI-QSM) is a highly sensible imaging technique to detect small amounts of non-heme ferric iron. It has been evaluated as an objective tool for detection of microbleeds associated with sentinel headache, and identification of high-risk intracranial aneurysms without radiological evidence of aSAH (figure 6). Our group published the first case report of micro-hemorrhage detection with 7T MRI-QSM in a patient with a $7 \mathrm{~mm}$ ACOM aneurysm, sentinel headache, and a negative CT/lumbar puncture. ${ }^{43} \mathrm{~A}$ follow-up study of 16 patients with 20 intracranial aneurysms demonstrated high correlation between positive MRI-QSM microbleeds, sentinel hemorrhage, and high aneurysm undulation index (undulation index=degree of surface concavity, which can vary with irregular domes or the presence of daughter sacs). ${ }^{44} 45$ MRI-QSM may be used to evaluate the presence of microbleeds in the aneurysm wall and surrounding tissues in patients with negative aSAH on CT/lumbar puncture or in 


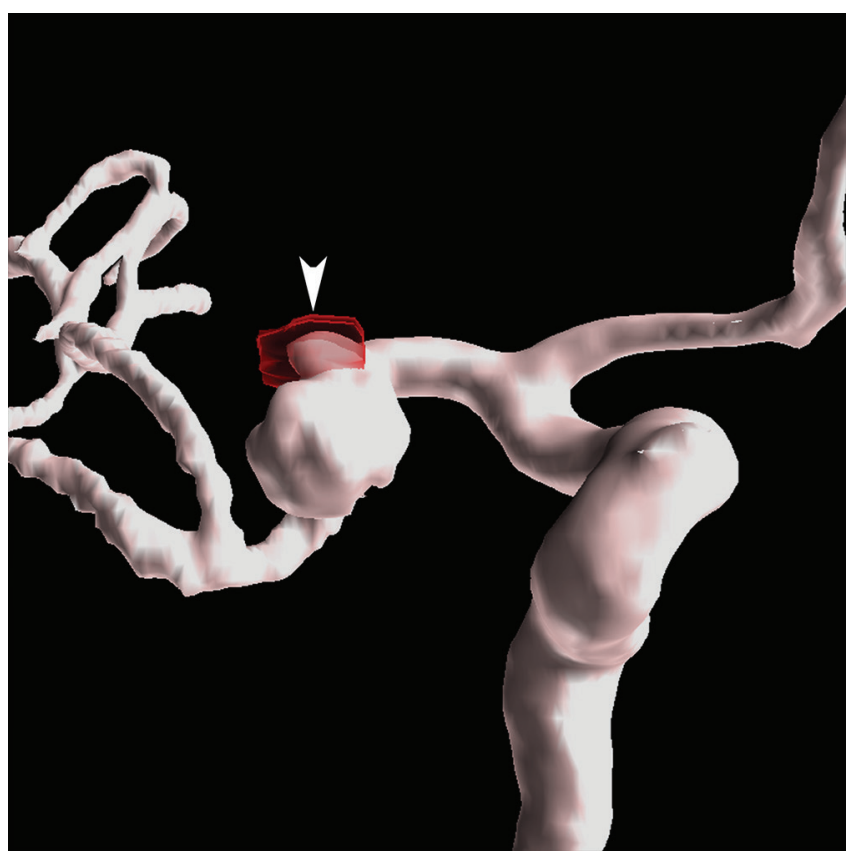

Figure 6 MRI-QSM 3D reconstruction image demonstrating a microhemorrhage (arrowhead) surrounding the daughter sac of the right middle cerebral artery aneurysm of a patient with sentinel headache.

patients with multiple aneurysms. One of the main limitations of MRI-QSM is that aneurysms located close to the skull base may not be well visualized due to bone artifact.

\section{7-TESLA MRI OF BRAIN ANEURYSMS}

7-Tesla HR-VWI is a powerful imaging technique in the assessment of intracranial aneurysms (table 2). Wrede et al prospectively evaluated 7 T time-of-flight (TOF) MRA and non-contrast enhanced magnetization-prepared rapid acquisition gradient-echo (MPRAGE) versus 1.5T TOF MRA for characterization of 20 UIAs. 7T MPRAGE imaging was superior to 1.5 and 7T TOF MRA in the assessment of aneurysm dome, neck, parent vessel, artifacts, vessel-tissue contrast, and overall image quality features. ${ }^{46}$ This group also demonstrated an almost perfect correlation between 7T MPRAGE/TOF MRA 2D/3D reconstructions and digital substraction angiography for identification and delineation of 64 UIAs (kappa 0.81-0.98). ${ }^{47}$

7T HR-VWI has been used for detailed anatomic characterization of the aneurysmal wall. Kleinloog et al studied 33 UIAs with 7T MRI T1-weighted 3D reconstructions. In vivo, the aneurysm wall and signal intensity variations were visualized in 28 of the 33 UIAs. Of note, because of partial volume effects, the authors inferred wall thickness variations in vivo MRIs using isotropic voxels instead of a high in-plane resolution, and validated their findings with both a wedge phantom and an ex vivo study. Ex vivo MRI showed variation in signal intensity across the aneurysm wall similar to the in vivo imaging. Signal intensity and wall thickness in both aneurysm wall specimens had a linear correlation (Pearson correlation coefficients of 0.85 and 0.86$).{ }^{48}$ Matsushige and colleagues used 7T TOF MRA and susceptibility-weighted imaging (SWI) to identify the triple-layered microstructure of the vessel wall of seven giant UIAs. The spatial resolution achieved in this study allowed visualization of in vivo microstructures. Moreover, histopathological analysis of two specimens revealed a homogeneous deposition of iron within the adventitia and smooth muscle layers. ${ }^{40}$ Because of a strong blooming effect in SWI at ultra-high magnetic field strengths, aneurysm wall thickness was overestimated approximately 1.5 -fold compared with TOF-MRA, which showed excellent correlation with histopathological measurements. Blankena and colleagues used 7T magnetization-prepared inversion-recovery turbo spin-echo (MPIR-TSE) sequences to correlate wall thickness and wall shear stress in 11 UIAs. The study showed a significant inverse association between apparent wall thickness and wall shear stress (mean correlation coefficient $-0.35, \mathrm{p}<0.05$ ), suggesting that 7T MPIR-TSE could be used to assess spatial wall thickness variations in relation to pathophysiologic processes such as aneurysm growth and rupture. ${ }^{49}$ These previous studies, and our experience with 7T imaging (unpublished material), demonstrate that the high spatial resolution achieved with 7T allows detailed characterization of the aneurysm wall and better sampling of signal intensity for determination of wall enhancement.

Table 2 HR-VWI of intracranial aneurysms on 7T scans

\begin{tabular}{|c|c|c|c|c|}
\hline Author (year) & Sample size & Imaging technique & VWI enhancement analysis & Findings \\
\hline Wrede $(2014)^{45}$ & 20 UIA & $\begin{array}{l}\text { 7T MRI TOF MRA/MPRAGE vs } \\
\text { 1.5T TOF MRA }\end{array}$ & $\begin{array}{l}\text { Non-contrast enhanced } \\
\text { MPRAGE }\end{array}$ & $\begin{array}{l}\text { 7T MPRAGE imaging was superior over } 1.5 \mathrm{~T} \text { and } 7 \mathrm{~T} \text { TOF MRA in the } \\
\text { assessment of aneurysm dome, neck, parent vessel, artifacts, vessel- } \\
\text { tissue contrast, and image quality }\end{array}$ \\
\hline Kleinloog $(2014)^{47}$ & 33 UIA & $\begin{array}{l}\text { 7T MRI TOF MRA and T1- } \\
\text { weighted 3D MPIR-TSE }\end{array}$ & $\begin{array}{l}\text { Compare with a wedge } \\
\text { phantom and two aneurysm } \\
\text { wall specimens }\end{array}$ & $\begin{array}{l}\text { Aneurysm wall SI variations were visualized in } 28 / 33 \text { UIAs } \\
\text { Strong correlation between aneurysm wall SI and histological } \\
\text { thickness (Pearson coefficient }=0.85 \text { ) }\end{array}$ \\
\hline Blankena $(2016)^{48}$ & 11 UIA & 7T MRI MPIR-TSE & $\begin{array}{l}\text { Correlate wall thickness and } \\
\text { wall shear stress }\end{array}$ & $\begin{array}{l}\text { Inverse association between apparent wall thickness and wall shear } \\
\text { stress (correlation coefficient }-0.35, \mathrm{p}<0.05 \text { ) }\end{array}$ \\
\hline Matsushige $(2016)^{40}$ & 7 UIA & 7T TOF MRA and SWI & $\begin{array}{l}\text { Triple-layered aneurysmal } \\
\text { vessel wall microstructure } \\
\text { analysis }\end{array}$ & $\begin{array}{l}\text { Increased iron deposits in the aneurysmal vessel wall from two } \\
\text { specimens, good correlation with TOF }\end{array}$ \\
\hline Wrede $(2017)^{46}$ & 64 UIA & $\begin{array}{l}\text { 7T MRI TOF MRA/MPRAGE vs } \\
\text { DSA }\end{array}$ & $\begin{array}{l}\text { Non-contrast enhanced } \\
\text { MPRAGE (2D and 3D } \\
\text { reconstructions) }\end{array}$ & $\begin{array}{l}\text { Correlation between 7T MRI 2D/3D reconstructions and DSA for } \\
\text { characterization of aneurysm dome, neck, parent vessel, artifacts, and } \\
\text { image quality (kappa } 0.81-0.98 \text { ) }\end{array}$ \\
\hline Wadghiri $(2018)^{49}$ & $\begin{array}{l}\text { Tissue from } \\
\text { clipped } \\
\text { aneurysms }\end{array}$ & Ultra HR-VWI 7T MRI & $\begin{array}{l}\text { Myeloperoxidase-gadolinium } \\
\text { chelate in phantoms and } \\
\text { human histology setups }\end{array}$ & $\begin{array}{l}\text { Neutrophil activity in the aneurysm wall and perivascular space } \\
\text { Active involvement of neutrophils and neutrophil extracellular traps }\end{array}$ \\
\hline
\end{tabular}

Gray rows highlight studies with histological correlate.

DSA, digital substraction angiography; HR-VWI, high-resolution vessel wall imaging; MPIR-TSE, magnetization-prepared inversion-recovery turbo spin-echo; MPRAGE, magnetization-prepared rapid acquisition gradient-echo; MRA, magnetic resonance angiography; MRI, magnetic resonance imaging; SI, signal intensity; SWI, susceptibilityweighted imaging; TOF, time-of-flight. 

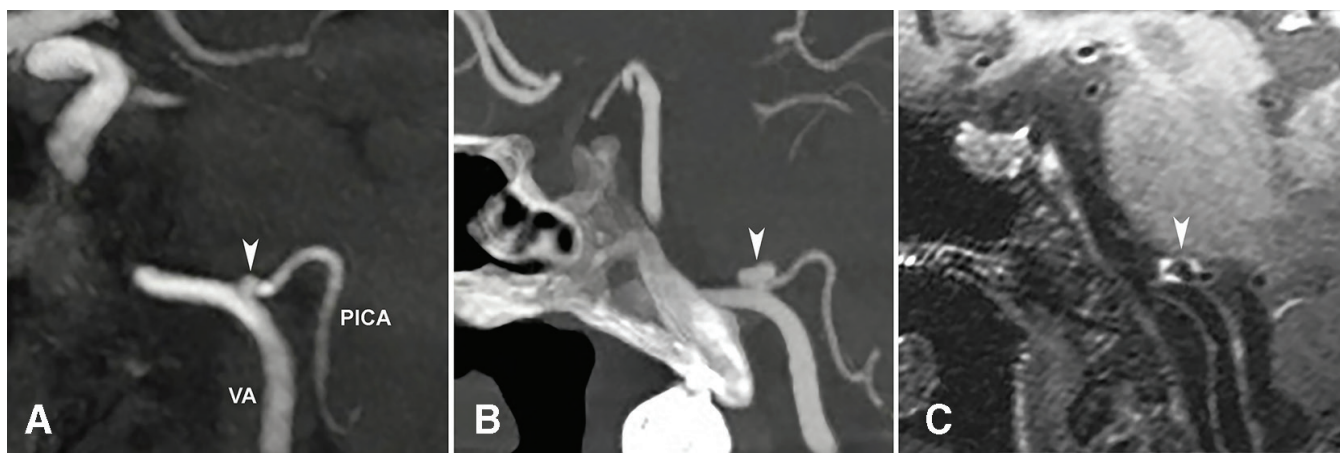

Figure 7 (A) Initial MR angiogram showing a small proximal posterior inferior cerebellar artery (PICA) aneurysm (arrowheads). (B) Twelve-month follow-up CT angiogram demonstrating enlargement of the PICA aneurysm and change in morphology (bilobed). (C) Post-contrast T1-weighted 7T MRI showing avid contrast enhancement of the aneurysm wall, suggestive of active inflammation .

Wadghiri et al tested MPO activity of the vessel wall of clipped aneurysms with 7T ultra HR-VWI MRI. ${ }^{50}$ This study tested a novel MPO-gadolinium chelate in a $\mu$ MRI histology setup and confirmed active neutrophil activity in the aneurysm wall and perivascular space. ${ }^{50}$ This novel study not only demonstrated that visualization of targeted immunological response is possible in brain aneurysms, but also tested a new MPO-gadolinium chelate for aneurysm imaging.

Although 7T MRI of intracranial aneurysms is still in development, it has shown promising results for characterization of aneurysm wall thickness, wall layering, and contrast enhancement. Our preliminary data suggest that 7T HR-VWI allows better detection of small aneurysm wall enhancement compared with conventional 3T scans (figure 7). Moreover, focal enhancement otherwise unnoticed with $3 \mathrm{~T}$ may be detected with 7T HR-VWI (figure 3).

\section{GAPS AND FUTURE RESEARCH}

Current evidence regarding the clinical use of HR-VWI in patients with intracranial aneurysms is limited. All available studies have an observational design, and no study has performed longitudinal follow-up of UIAs over time. Thus, HR-VWI has not been validated in any clinical study and may not be used consistently for clinical decisions. However, HR-VWI is being used nowadays as a surrogate marker of aneurysm recurrence and progression after endovascular treatment. Zhang et al analyzed 83 dissecting vertebrobasilar aneurysms with $3 \mathrm{~T}$ HR-VWI and determined that wall enhancement and aneurysm diameter were independent factors associated with recurrence $(\mathrm{p}<0.05) .^{51}$

There is significant heterogeneity among the HR-VWI sequences and protocols used for assessment of intracranial aneurysms. Slice thickness, flip angle, repetition/echo times, field/matrix/voxel sizes, and scan times have not been standardized, thus limiting its reproducibility in daily practice. ${ }^{8}$ Moreover, as previously discussed, flow artifact, contrast extravasation, and stagnant flow could mimic wall enhancement on HR-VWI. ${ }^{52}$

Furthermore, there is no consensus on the standard definition of wall enhancement. Some studies have evaluated only 'circumferential' wall enhancement while others have included aneurysms with 'partial' wall enhancement. Most studies classified enhancement intensity subjectively into different qualitative categories—strong/avid enhancement versus faint or no enhancement-instead of using more reproducible quantitative imaging techniques. Other studies have used objective data generated from the quantification of signal intensity in the aneurysm wall. To decrease variability, a signal intensity ratio is generated through comparison with the pituitary stalk ${ }^{29}$ or pre- and post-gadolinium T1-weighted sequences. ${ }^{32}$ Because of all these limitations, the extent to which aneurysm wall enhancement can be considered an independent biomarker for aneurysm instability remains controversial. A multicenter prospective registry with a standardized and validated imaging protocol is required to use HR-VWI reliably in clinical decisions.

We and others have identified the following gaps of HR-VWI in the characterization of UIAs ${ }^{53}$ :

- There is no accurate definition of aneurysm instability: growth, change of morphology, sentinel headache and/or cranial nerve mass effect. Rupture is the endpoint of aneurysm instability and may not be used to validate HR-VWI.

- Lack of standardization of wall enhancement. Every study has used a different method to establish wall enhancement. An objective and automated method should be validated among different scans.

- Lack of consistency in the histological correlate of aneurysm enhancement.

\section{CONCLUSION}

Current evidence suggests that HR-VWI could potentially provide new insights in the management of intracranial aneurysms. As imaging techniques continue to improve, HR-VWI may become an important non-invasive biomarker of aneurysm instability and risk of rupture. Data suggest that the lack of aneurysm wall enhancement has a strong negative predictive value. Paucity of prospectively validated data limits the clinical use of HR-VWI in treatment decisions of UIAs. As such, a prospective multicenter trial of HR-VWI as a biomarker of aneurysm instability is needed. Such a trial will define if aneurysms $\leq 7 \mathrm{~mm}$ in size without wall enhancement should only be observed as they pose a small risk of rupture.

Contributors Conception, design and study supervision: EAS. All named authors contributed substantially to the work described by actively participating in the study and the generation of the data, and provided critical evaluation of the manuscript.

Funding The authors have not declared a specific grant for this research from any funding agency in the public, commercial or not-for-profit sectors.

Competing interests None declared.

Patient consent for publication Not required.

Provenance and peer review Commissioned; externally peer reviewed. 
ORCID iD

Edgar A Samaniego http://orcid.org/0000-0003-2764-2268

\section{REFERENCES}

1 Vlak MH, Algra A, Brandenburg R, et al. Prevalence of unruptured intracranial aneurysms, with emphasis on sex, age, comorbidity, country, and time period: a systematic review and meta-analysis. Lancet Neurol 2011;10:626-36.

2 Karamanakos PN, von Und Zu Fraunberg M, Bendel S, et al. Risk factors for three phases of 12-month mortality in 1657 patients from a defined population after acute aneurysmal subarachnoid hemorrhage. World Neurosurg 2012;78:631-9.

3 Bijlenga P, Gondar R, Schilling S, et al. PHASES score for the management of intracranial aneurysm: a cross-sectional population-based retrospective study. Stroke 2017:48:2105-12

4 Samaniego EA, Roa JA, Martinez-Burbano B, et al. Angiographic features of intracranial aneurysms in Ecuador. J Stroke Cerebrovasc Dis 2019;28:761-7.

5 Texakalidis $\mathrm{P}$, Hilditch CA, Lehman V, et al. Vessel wall imaging of intracranial aneurysms: systematic review and meta-analysis. World Neurosurg 2018;117:453-8.

6 Hudson JS, Hoyne DS, Hasan DM. Inflammation and human cerebral aneurysms: current and future treatment prospects. Future Neurol 2013;8:663-76.

7 Virchow R. Ueber die akute Entzündung der Arterien. Virchows Arch A Pathol Anat Histopathol 1847;1:272-378.

8 Lehman VT, Brinjikji W, Mossa-Basha M, et al. Conventional and high-resolution vessel wall MRI of intracranial aneurysms: current concepts and new horizons. J Neurosurg 2018:128:969-81.

9 Tulamo R, Frösen J, Hernesniemi J, et al. Inflammatory changes in the aneurysm wall: a review. J Neurointerv Surg 2018;10(Suppl 1):i58-67.

10 Chyatte D, Bruno G, Desai S, et al. Inflammation and intracranial aneurysms. Neurosurgery 1999;45:1137-47.

11 Chalouhi N, Ali MS, Jabbour PM, et al. Biology of intracranial aneurysms: role of inflammation. J Cereb Blood Flow Metab 2012;32:1659-76.

12 Crompton MR. Mechanism of growth and rupture in cerebral berry aneurysms. Br Med J 1966;1:1138-42.

13 Hu P, Yang Q, Wang DD, et al. Wall enhancement on high-resolution magnetic resonance imaging may predict an unsteady state of an intracranial saccular aneurysm. Neuroradiology 2016;58:979-85.

14 Larsen N, von der Brelie C, Trick D, et al. Vessel wall enhancement in unruptured intracranial aneurysms: an indicator for higher risk of rupture? High-resolution MR imaging and correlated histologic findings. AJNR Am I Neuroradiol 2018;39:1617-21

15 Shimonaga K, Matsushige T, Ishii D, et al. Clinicopathological insights from vessel wall imaging of unruptured intracranial aneurysms. Stroke 2018;49:2516-9.

16 Hudson IS, Zanaty M, Nakagawa D, et al. Magnetic resonance vessel wall imaging in human intracranial aneurysms. Stroke 2018 [Epub ahead of print].

17 Matsushige T, Shimonaga K, Mizoue T, et al. Focal aneurysm wall enhancement on magnetic resonance imaging indicates intraluminal thrombus and the rupture point. World Neurosurg 2019 [Epub ahead of print].

18 Quan K, Song J, Yang Z, et al. Validation of wall enhancement as a new imaging biomarker of unruptured cerebral aneurysm. Stroke 2019;50:1570-3.

19 Chalouhi N, Hoh BL, Hasan D. Review of cerebral aneurysm formation, growth, and rupture. Stroke 2013;44:3613-22.

20 Lv N, Karmonik C, Chen S, et al. Relationship between aneurysm wall enhancement in vessel wall magnetic resonance imaging and rupture risk of unruptured intracranial aneurysms. Neurosurgery 2019;84:E385-E391.

21 Hartman JB, Watase H, Sun J, et al. Intracranial aneurysms at higher clinical risk for rupture demonstrate increased wall enhancement and thinning on multicontrast 3D vessel wall MRI. Br J Radiol 2019;92:20180950.

22 Alexander MD, Yuan C, Rutman A, et al. High-resolution intracranial vessel wall imaging: imaging beyond the lumen. J Neurol Neurosurg Psychiatry 2016;87:589-97.

23 Backes D, Hendrikse J, van der Schaaf I, et al. Determinants of gadoliniumenhancement of the aneurysm wall in unruptured intracranial aneurysms. Neurosurgery 2018;83:719-25.

24 Liu P, Qi H, Liu A, et al. Relationship between aneurysm wall enhancement and conventional risk factors in patients with unruptured intracranial aneurysms: a blackblood MRI study. Interv Neuroradiol 2016;22:501-5.

25 Wang GX, Li W, Lei S, et al. Relationships between aneurysmal wall enhancement and conventional risk factors in patients with intracranial aneurysm: a high-resolution MR study. J Neuroradio/ 2019;46:25-8.

26 Liang L, Steinman DA, Brina O, et al. Towards the clinical utility of CFD for assessment of intracranial aneurysm rupture - a systematic review and novel parameter-ranking tool. J Neurointerv Surg 2019;11:153-8.

27 Cantrell CG, Vakil P, Jeong Y, et al. Diffusion-compensated tofts model suggests contrast leakage through aneurysm wall. Magn Reson Med 2017;78:2388-98.
28 Edjlali M, Gentric JC, Régent-Rodriguez C, et al. Does aneurysmal wall enhancement on vessel wall MRI help to distinguish stable from unstable intracranial aneurysms? Stroke 2014;45:3704-6.

29 Omodaka S, Endo H, Niizuma K, et al. Circumferential wall enhancement in evolving intracranial aneurysms on magnetic resonance vessel wall imaging. J Neurosurg 2018:1-7 [Epub ahead of print].

30 Nagahata S, Nagahata M, Obara M, et al. Wall enhancement of the intracranial aneurysms revealed by magnetic resonance vessel wall imaging using threedimensional turbo spin-echo sequence with motion-sensitized driven-equilibrium: a sign of ruptured aneurysm? Clin Neuroradiol 2016;26:277-83.

31 Edjlali M, Guédon A, Ben Hassen W, et al. Circumferential thick enhancement at vessel wall MRI has high specificity for intracranial aneurysm instability. Radiology 2018:289:181-7.

32 Wang GX, Gong MF, Zhang D, et al. Wall enhancement ratio determined by vessel wall MRI associated with symptomatic intracranial aneurysms. Eur J Radiol 2019;112:88-92

33 Nehls DG, Flom RA, Carter LP, et al. Multiple intracranial aneurysms: determining the site of rupture. J Neurosurg 1985;63:342-8.

34 Matouk CC, Mandell DM, Günel M, et al. Vessel wall magnetic resonance imaging identifies the site of rupture in patients with multiple intracranial aneurysms: proof of principle. Neurosurgery 2013;72:492-6.

35 Coutinho JM, Sacho RH, Schaafsma JD, et al. High-resolution vessel wall magnetic resonance imaging in angiogram-negative non-perimesencephalic subarachnoid hemorrhage. Clin Neuroradiol 2017;27:175-83.

36 Backes D, Vergouwen MD, Velthuis BK, et al. Difference in aneurysm characteristics between ruptured and unruptured aneurysms in patients with multiple intracranial aneurysms. Stroke 2014;45:1299-303.

37 Kondo R, Yamaki T, Mouri W, et al. [Magnetic resonance vessel wall imaging reveals rupture site in subarachnoid hemorrhage with multiple cerebral aneurysms]. No Shinkei Geka 2014;42:1147-50.

38 Mossa-Basha M, Huynh TJ, Hippe DS, et al. Vessel wall MRI characteristics of endovascularly treated aneurysms: association with angiographic vasospasm. $J$ Neurosurg 2018:1-9. [Epub ahead of print].

39 Wang $X$, Zhu $C$, Leng $Y$, et al. Intracranial aneurysm wall enhancement associated with aneurysm rupture: a systematic review and meta-analysis. Acad Radiol 2019;26.

40 Matsushige T, Chen B, Ringelstein A, et al. Giant intracranial aneurysms at 7T MRI. AJNR Am J Neuroradiol 2016;37:636-41.

41 Honkanen P, Frösen JK, Abo-Ramadan U, et al. Visualization of luminal thrombosis and mural Iron accumulation in giant aneurysms with ex vivo 4.7T magnetic resonance imaging. Surg Neurol Int 2014;5:74.

42 Hasan D, Chalouhi N, Jabbour P, et al. Early change in ferumoxytol-enhanced magnetic resonance imaging signal suggests unstable human cerebral aneurysm: a pilot study. Stroke 2012;43:3258-65.

43 Nakagawa D, Cushing C, Nagahama Y, et al. Quantitative susceptibility mapping as a possible tool to radiographically diagnose sentinel headache associated with intracranial aneurysm: case report. World Neurosurg 2017;103:954.e1-954.e4.

44 Nakagawa D, Kudo K, Awe O, et al. Detection of microbleeds associated with sentinel headache using MRI quantitative susceptibility mapping: pilot study. J Neurosurg 2018:1-7.

45 Dhar S, Tremmel M, Mocco J, et al. Morphology parameters for intracranial aneurysm rupture risk assessment. Neurosurgery 2008;63:185-97.

46 Wrede KH, Dammann P, Mönninghoff $C$, et al. Non-enhanced MR imaging of cerebral aneurysms: 7 Tesla versus 1.5 Tesla. PLoS One 2014;9:e84562.

47 Wrede KH, Matsushige T, Goericke SL, et al. Non-enhanced magnetic resonance imaging of unruptured intracranial aneurysms at 7 Tesla: comparison with digital subtraction angiography. Eur Radiol 2017;27:354-64.

48 Kleinloog R, Korkmaz E, Zwanenburg JJ, et al. Visualization of the aneurysm wall: a 7.0-tesla magnetic resonance imaging study. Neurosurgery 2014;75:614-22.

49 Blankena R, Kleinloog R, Verweij BH, et al. Thinner regions of intracranial aneurysm wall correlate with regions of higher wall shear stress: a 7T MRI study. AJNR Am J Neuroradiol 2016:37:1310-7.

50 Wadghiri YZ, Hoang DM, Leporati A, et al. High-resolution imaging of myeloperoxidase activity sensors in human cerebrovascular disease. Sci Rep 2018:8:7687.

51 Zhang Y, Sui B, Liu J, et al. Aneurysm wall enhancement on magnetic resonance imaging as a risk factor for progression of unruptured vertebrobasilar dissecting aneurysms after reconstructive endovascular treatment. J Neurosurg 2018:128:747-55.

52 Kalsoum E, Chabernaud Negrier A, Tuilier T, et al. Blood flow mimicking aneurysmal wall enhancement: a diagnostic pitfall of vessel wall MRI using the postcontrast 3D turbo spin-echo MR imaging sequence. AJNR Am I Neuroradiol 2018:39:1065-7.

53 Lehman VT, Brinjikji W. Vessel wall imaging of unruptured intracranial aneurysms: ready for prime time? Not so fast!. AJNR Am J Neuroradiol 2019:40:E26-9. 